\title{
Simulation of Two Stages Cascode LNA Using Ladder Matching Networks for WiMAX Applications
}

\author{
A.B. Ibrahim \\ Faculty of Art \\ Computing and Creative Industry \\ Universiti Pendidikan Su ltan Idris \\ Malaysia
}

\begin{abstract}
This paper presents a simulation of two stages Cascode LNA using ladder matching network applicable for WiMAX applications. It's using the FHX76LP Low Noise SuperHEMT FET transistor. The LNA designed used Ladder-matching network consisting of lump reactive element at the input and the T-matching network at output terminal. The cascode low noise amplifier (LNA) produced gain of $38.93 \mathrm{~dB}$ and noise figure (NF) of 1.12 dB. The input reflection (S11) and output return loss (S22) are $-18.11 \mathrm{~dB}$ and $-\mathbf{2 2 . 1 4} \mathrm{dB}$ respectively. The bandwidth of the amplifier recorded is $1.5 \mathrm{GHz}$. The input sensitivity is compliant with the IEEE 802.16d standards.
\end{abstract}

Keyword-cascode LNA; radio frequency; ladder -matching network

\section{INTRODUCTION}

The market of wire less communications system is growing rapidly. Due to market demands, there exist various wireless communication systems at different frequency band for different applications. WiMAX, which is short for Worldwide Interoperability for Microwave Access, is a novel wireless communication technology. It is an attractive technology due to the high transmitting speed (70 Mbps) and long transmitting distance $(50 \mathrm{Km})$. This system is based on IEEE 802.16 standard and uses several bands $(2.3-2.7 \mathrm{GHz}, 3.4-3.6 \mathrm{GHz}$, and $5.1-5.8 \mathrm{GHz}$ ) to transmit data. The design of the front-end low noise amplifier (LNA) is one of the challenges in Radio Frequency (RF) receiver. A good LNA needs to provide good input impedance match, enough power gain and low noise figure (NF) within the required band [1].

WiMAX, which is short for Worldwide Interoperability for Microwave Access, is a novel wireless communication technology. It is an attractive technology due to the high transmitting speed (up to $70 \mathrm{Mbps}$ ) and long transmitting distance (up to 30 mile). The system bases on IEEE 802.16 standards and uses several bands $(2.3-2.7 \mathrm{GHz}, 3.4-3.6 \mathrm{GHz}$ and $5.1-5.8 \mathrm{GHz}$ ) to transmit data. The design of the front-end low noise amplifier (LNA) is one of the challenges in radio frequency $(\mathrm{RF})$ receivers, which needs to provide good input impedance match, enough power gain and low noise figure (NF) within the required band [2] [8].Many high gain amplifier topologies have been proposed as a way to satisfy the requirement for low power dissipation as well as good performances. The cascode techniques to produces results in a higher bandwidth and gain, due to the increase in the output impedance, as well as better isolation between the input and output ports [3] [7].

\section{THEORETICAL ASPECTS}

Initially, when designing an amplifier, the input and output matching network are consider to achieve the required stability, small signal gain, and bandwidth. Super high frequency amplifier is a typical active circuit used to amplify the amplitude of RF signal. Basic concept and consideration in design of super high frequency amplifier is presented in this paper. The LNA designed, the formula and equation were referred to [4]. Figure 1, shows a typical single-stage amplifier including input/output matching networks. The basic concept of high frequency amplifier design is to match input/output of a transistor at high frequencies using S-parameters frequency characteristics at a specific DC-bias point with source impedance and load impedance. Input/output matching circuit is essential to reduce the unwanted reflection of signal and to improve efficiency of the transmission from source to load [4], [5].

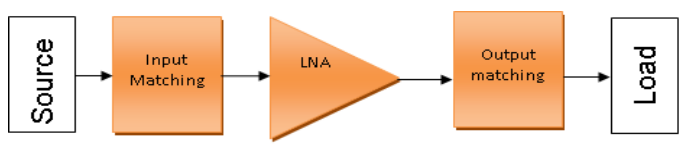

FIGURE I. TYPICAL AMPLIFIER DESIGN.

\section{A. Power Gain}

Several power gains were defined in order to understand operation of super high frequency amplifier. Figure 2, show that power gains of 2-port circuit network with power impedance or load impedance at power amplifier. The power amplifiers represented with scattering coefficients are clas sified into Operating Power Gain, Transducer Power Gain and Available Power Gain [4] [5].

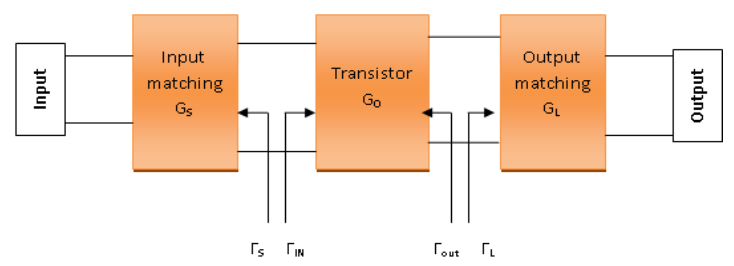

FIGURE II. I/O CIRCUIT OF 2-PORT NETWORK.

\section{B. Operating Power Gain}

Operating power gain is the ratio of load power $\left(\mathrm{P}^{L}\right)$ delivered to the load $\left(\mathrm{Z}^{L}\right)$ to input power $\left(\mathrm{P}^{\text {in }}\right)$ supplied to 2- 
port network. Power delivered to the load is the difference between the power reflected at the output port and the input power, and power supplied to 2-port network is the difference between the input power at the input port and the reflected power. Therefore, Operating Power Gain is represented by

$$
\begin{aligned}
& G_{P}=\frac{\text { Power delivered to the load }}{\text { power supplied to the amplifier }} \\
& =\frac{P_{L}}{P_{\text {in }}}=\frac{1}{1-\left|\Gamma_{\text {in }}\right|^{2}}\left|S_{21}\right|^{2} \frac{1-\left|\Gamma_{L}\right|^{2}}{\left|1-S_{22} \Gamma_{L}\right|^{2}}
\end{aligned}
$$

Where, $\Gamma_{\text {in }}$ indicates reflection coefficient of load at the input port of 2-port network and $\Gamma_{s}$ is reflection coefficient of power supplied to the input port.

\section{Transducer Power Gain}

Transducer Power Gain is the ratio of $P_{a v s}$, maximum power available from source to $P_{L}$, power delivered to the load. As maximum power is obtained when input impedance of circuit network is equal to conjugate comple $\mathrm{x}$ number of power impedance, if $\Gamma_{i n}=\Gamma_{s}$, transducer power gain is represented by

$$
\begin{aligned}
& G_{T}=\frac{\text { Power delivered to the load }}{\text { Power Available from the source }} \\
& =\frac{P_{L}}{P_{\text {avs }}}=\frac{\left|S_{21}\right|^{2}\left(1-\left|\Gamma_{S}\right|^{2}\right)\left(1-\left|\Gamma_{L}\right|^{2}\right)}{\left|\left(1-S_{11} \Gamma_{S}\right)\left(1-S_{22} \Gamma_{L}\right)-\left(S_{12} S_{21} \Gamma_{S} \Gamma_{L}\right)\right|^{2}}
\end{aligned}
$$

Where, $\Gamma_{L}$ indicates load reflection coefficient.

\section{Available Power Gain}

Available Power Gain, $G_{A}$ is the ratio of $P_{a v s}$, power available from the source, to $P_{\text {avn }}$, power available from 2-port network, that is, $G_{A}=\frac{P_{a v n}}{P_{a v s}}$. Power gain is $P_{a v n}$ when $\Gamma_{\text {in }}=\Gamma^{*}{ }_{s}$. Therefore Available Power Gain is given by:

$$
\begin{aligned}
G_{A} & =\frac{\text { Power available from the amplifier }}{\text { Power available from the source }} \\
& =\frac{P_{a v n}}{P_{a v s}}=\frac{1-\left|\Gamma_{S}\right|^{2}}{\left|1-S_{11} \Gamma_{S}\right|^{2}}\left|S_{21}\right|^{2} \frac{1}{\left|1-S_{22} \Gamma_{L}\right|^{2}}
\end{aligned}
$$

That is, the above formula indicates power gain when input and output are matched [5].

\section{E. Noise Figure}

Signals and noises applied to the input port of amplifier were amplified by the gain of the amplifier and noise of amplifier itself is added to the output. Therefore, SNR (Signal to Noise Ratio) of the output port is smaller than that of the input port. The ratio of SNR of input port to that of output port is referred to as noise figure and is larger than $1 \mathrm{~dB}$. Typically, noise figure of 2-port transistor has a minimum value at the specified ad mittance given by formula:

$$
F=F_{\text {min }}+\frac{R_{N}}{G_{S}}\left|Y_{s}-Y_{o p t}\right|^{2}
$$

Where, $\mathrm{RN}$ is the equivalent noise resistance of two ports. Fmin is the minimum noise factor obtained by adjusting tuners at the input of the amplifier. The normalized presented by the tuners at Fmin is Yopt. With Ys $=$ Ys/Zo being the actual normalized admittance.

For low noise transistors, manufactures usually provide $F_{\min }, R_{N}, Y_{o p t}$ by frequencies. $\mathrm{N}$ defined by formula for desired noise figure:

$$
N=\frac{\left|\Gamma_{s}-\Gamma_{o p t}\right|^{2}}{1-\left|\Gamma_{S}\right|^{2}}=\frac{F-F_{\min }}{4 R_{N} / Z_{0}}\left|1+\Gamma_{o p t}\right|^{2}
$$

\section{F. Condition for Matching}

The scattering coefficients of transistor were determined. The only fle xibility permitted to the designer is the input/output matching circuit. The input circuit should match to the source and the output circuit should match to the load in order to deliver maximum power to the load. After stability of active device is demand, input/output matching circuits should be designed so that reflection coefficient of each port is correlated with conjugate comple x number as given below [6]:

$$
\begin{gathered}
\Gamma_{I N}=\Gamma_{S}^{*}=S_{11}+\frac{S_{12} S_{21} \Gamma_{L}}{1-S_{22} \Gamma_{L}} \\
\Gamma_{\text {oUT }}=\Gamma_{L}^{*}=S_{22}+\frac{S_{12} S_{21} \Gamma_{S}}{1-S_{11} \Gamma_{S}}
\end{gathered}
$$

The noise figure of the first stage of the receiver overrules noise figure of the whole system. To get a minimum noise figure using a transistor, power reflection coefficient should match with $\Gamma_{\text {opt }}$ and load reflection coefficient should match with $\Gamma_{\text {out }}^{*}$

$$
\begin{aligned}
\Gamma_{s} & =\Gamma_{\text {opt }} \\
\Gamma_{L} & =\Gamma_{\text {out }}^{*}=\left(S_{22}+\frac{S_{12} S_{21} \Gamma_{s}}{1-S_{11} \Gamma_{s}}\right)
\end{aligned}
$$

\section{DESIGN OF LNA}

The proposed topology, shown in Fig. 3(a) and (b) uses a ladder matching network for input matching network port, while the T-matching network uses at the output port respectively to provider the good performance in term of stability, power gain and S-Parameter. The circuit of matching techniques is simulated using Advance Design System to fine tune and further optimization for better performance. 


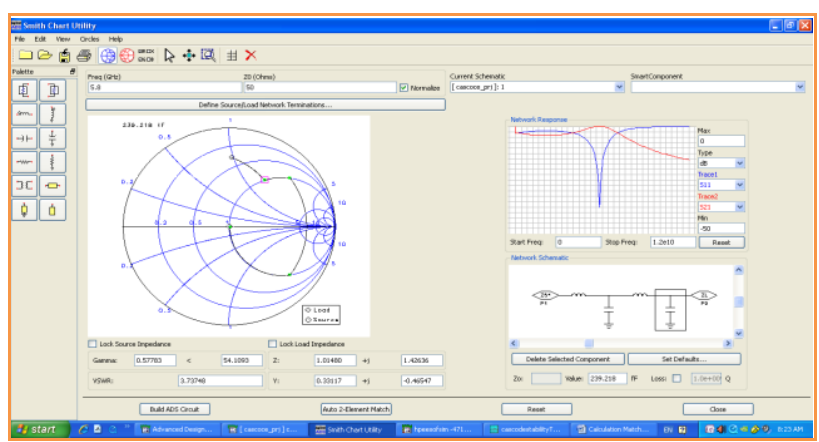

FIGURE III. (A):LADDER MAT CHING NETWORK FOR INPUT.

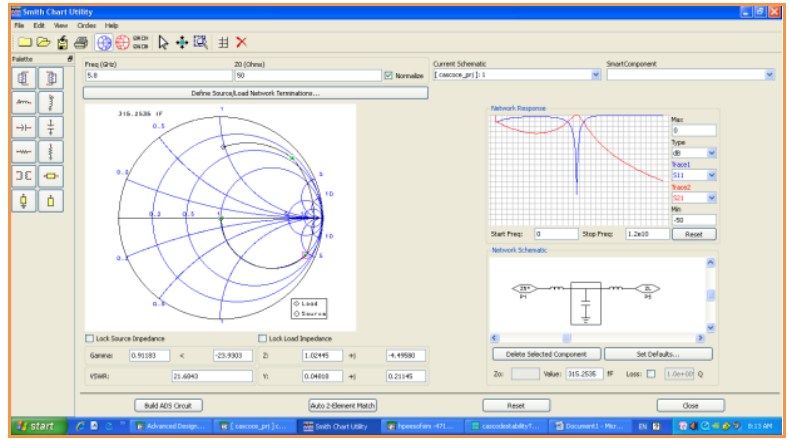

FIGURE III. (B): -MATCHING NETWORK FOR OUTPUT.

The goals in LNA design are to maximize its gain and minimize its noise figure with sufficient linearity and impedance matching [1]. In order to achieved the key demands for WiMAX receiver characteristics, a LNA is designed should be met are the noise figure less than $3 \mathrm{~dB}$ and power gain should be more than $20 \mathrm{~dB}$. Also good input and output impedance matching to achieved the s-parameter values.

Figure 3 shows, the complete schematic circuit of $5.8 \mathrm{GHz}$ a Cascode Low noise amplifier. It was simulated using the same software to fine and further optimized for a better performance. For purpose of fabrication, the inductances and capacitances need to be converted to microstrip layout. The Duriod 5880 TYL-0200 was selected for fabricate. The LNA parameter is shown in Table I.

TABLE I. LNAPARAMETERS.

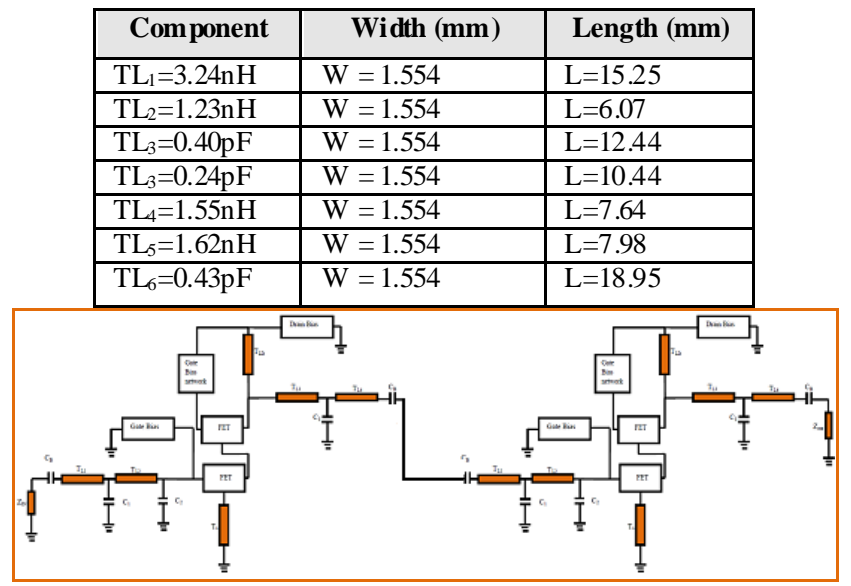

FIGURE IV. THE SCHEMATIC LAYOUT FOR TWO ST AGES CASCODE LNA.

\section{SIMULATION RESULT}

Table II shows the s-parameters output of Cascode LNA. It is simulated using Advanced Design System (ADS). The simulation recorded that the power gains $\mathrm{S} 21$ is $38.93 \mathrm{~dB}$. The input return loss $\mathrm{S} 11$ is $-18.11 \mathrm{~dB}$; overall noise figure (NF) of $1.12 \mathrm{~dB}$ and the output return loss $\mathrm{S} 22$ is $-22.14 \mathrm{~dB}$. The reflection loss $\mathrm{S} 12$ is $-44.28 \mathrm{~dB}$. These values were within the design specification and were accepted. The outputs Sparameter are shows in Figure 4a and 4b.Figure 4a shows the input and output return loss while, Figure $4 \mathrm{~b}$ shows the noise figure and the stability.

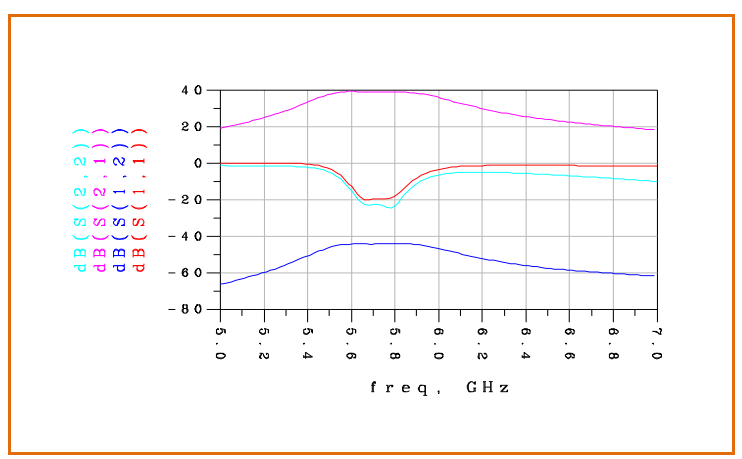

FIGURE V. (A) $\mathrm{S}_{21}, \mathrm{~S}_{12}, \mathrm{~S}_{22}$ AND $\mathrm{S}_{11}$

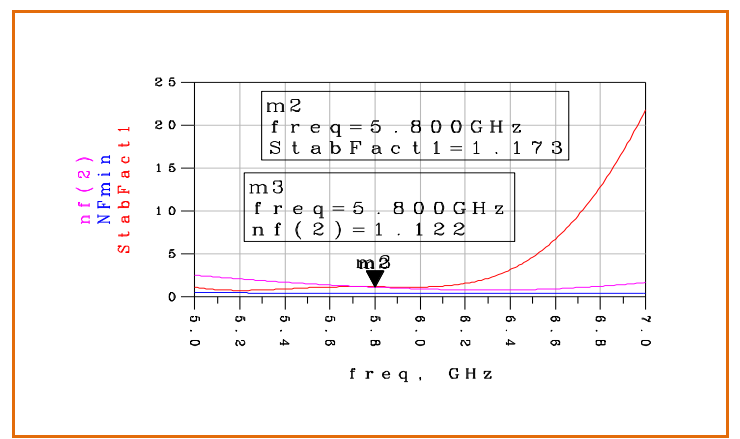

FIGURE V. (B): NOISE FIGURE AND ST ABILITY FACTOR (K).

TABLE II.SIMULATION RESULTS.

\begin{tabular}{|c|c|c|}
\hline S Parameters & Targe ted & Simulated \\
\hline Input Reflection $\mathrm{S}_{11} \mathrm{~dB}$ & $<-10 \mathrm{~dB}$ & -18.11 \\
\hline Return Loss $\mathrm{S}_{12} \mathrm{~dB}$ & $<-10 \mathrm{~dB}$ & -44.28 \\
\hline Forward transfer $\mathrm{S}_{21} \mathrm{~dB}$ & $>50 \mathrm{~dB}$ & 38.93 \\
\hline Output Reflection $\mathrm{S}_{22} \mathrm{~dB}$ & $<-10 \mathrm{~dB}$ & -22.14 \\
\hline $\mathrm{NF} \mathrm{dB}$ & $<3 \mathrm{~dB}$ & 1.12 \\
\hline $\mathrm{BW} \mathrm{MHz}$ & $>1000$ & 1500 \\
\hline
\end{tabular}

\section{CONCLUSION}

A two stages cascoded low noise amplifier with ladder matching techniques has been simulated and designed. The simulation results have to meet for $802.16 \mathrm{~d}$ standards for WiMAX applications. It is observed that the simulated and targeted results giving almost the same figure as required. It observed that the gain of the simulated analysis is $38.4 \mathrm{~dB}$. It is important to take note when designing the amplifier to match the amplifier circuits. The $5.8 \mathrm{GHz}$ LNA has been developed successfully and the circuit contributed to the front end receiver 
at the described frequency. For better performance in gain of the amplifier, it can be achieved by increasing the number of stages to improve the gain and noise figure of the design [9]. Higher gain would expand the coverage or communication distance.

\section{REFERENCES}

[1] Jelana Misic." Wideband Low Noise Amplifier for Long Term Evolution Systems ". Proceeding of the $5^{\text {th }}$ Systems Simulation Symposium 2014.

[2] Ruey-Lue Wang, Shih-Chih Chen,Cheng-Lin Huang,Chien-Hsuan Lie,Yi-Shu Lin. "2-6GHz Current-Reused LNA With Transformer-type Induct ors." IEEE Proceeding 2008.

[3] Leon, Michael Angelo G.Lorenzo and Maria Theresa G.De. "Comparison of LNA Topology for Wimax Application in a Standard 90-nm CMOS Process." 12th International Conference on Computer Modelling and Simulation. 2010. pp-642-647.

[4] M.Pozar, David. Microwave and RF Wireless System. Third Avenue,N.Y.John Wiley \& Sons, in 2001.

[5] Gonzalez, Guillermo. Microwave Transistor Amplifier. 1996.

[6] Othman A.R, Hamidon A.H,Ting J.T.H and Mustaffa M.F. "High Gain Cascaded Low Noise Amplifier Using T-Matching Net work." $4^{\text {th }}$ ISBC 2010.

[7] Weber, Wuezhan Wang and Robert. "Design of a CMOS Low Noise Amplifier (LNA) at $5.8 \mathrm{GHz}$ and its Sensitivity Analysis." $11^{\text {th }}$ NASA Symposium 2003.

[8] IEEE Computer Society and IEEE Microwave Theory Technique and Society.Part 16 Air Interface For Fix Broadband Wireless System, IEEE Standard 802.16.2004

[9] Inder J.Bahl. Fundamentals of RF and Microwave Transistor Amplifier. 2009 